\title{
A Case of Cervical Dystonia with Non Responsive To Usual Noninvasive Treatment
}

\author{
Hafez $\mathrm{F}^{1}$, Islam $\mathrm{MZ}^{2}$, Nessa $\mathrm{J}^{3}$, Shahidullah $\mathrm{MS}^{4}$, Sobhan $\mathrm{S}^{5}$, Begum $\mathrm{S}^{6}$
}

\begin{abstract}
Cervical dystonia is a focal dystonia characterized by neck muscles contracting involuntarily-causing abnormal movement and awkward posture of the head and neck. A rare disorder can occur at any age even at infancy. Symptoms generally begin gradually and then reach a point where they don't get substantially worse. Asymmetry of the sternocleidomastoid (SCM) is often present in untreated patient. There is no cure for cervical dystonia. Injecting botulinum toxin into the affected muscles often reduces the signs and symptoms of cervical dystonia. In this case, it has been described a rare case of a forty seven year old male patient with a history of cervical dystonia presented with struggle with head position, resisting but never overcoming the tendency of his head to assume an unnatural position. The management including drugs like oral analgesic, muscle relaxant, anti-Parkinson drug \& even antipsychotic drug were used. Physiatric approach like different modalities of heat such as Short wave diathermy (SWD), Microwave diathermy (MWD) \& Ultra sound therapy (UST) were used. Brace like cervical collar and different type of exercise was also prescribed, even botulinum toxin injected into suspected muscles but all discussed above failed in this case.

[J Shaheed Suhrawardy Med Coll, 2014;6(1):44-46]
\end{abstract}

Key Words: cervical dystonia, Sternocleidomastoid (SCM), botulinum toxin

Received: January 2014; Revised: March 2014; Accepted: April 2014

\section{Introduction}

Cervical dystonia also called spasmodic torticolis ${ }^{1}$ is a painful condition in which neck muscle contract involuntarily, causing head to twist or turn to one side, can also cause head to uncontrollably tilt forward or backward $^{2}$. Movement is a key finding in cervical dystonia. Another prominent finding is evidence of active muscle contraction in the form of muscle thickening and hypertrophy. The movement maybe sustained (tonic), jerky (clonic) or a combination ${ }^{3}$. Spasms in the muscles or pinched nerve in the neck can result in considerable pain and discomfort and expanded neck size can occur.
Asymmetry of Sternocleidomastoid muscle is often present in untreated patient. The condition appears sporadically in the absence of a documented family history of the disease. In about $10-15 \%$ of cases more than one family member maybe affected. Several families have been attributed with autosomal dominant ${ }^{4}$, adult onset, primary dystonia. That is focal in distribution affecting the neck region. This form of condition has been called familial torticollis. Cervical dystonia may be primary (meaning that it is the only apparent neurological disorder with or without a family history) or be brought about by secondary ${ }^{3,4}$ causes such as physical trauma. Cases of inherited cervical dystonia may

1. Dr. Fahmida Hafez, Assistant Professor, Department of Physical Medicine \& Rehabilitation, National Institute of Cardiovascular Disease, Dhaka

2. Dr. Md. Zahidul Islam, Assistant Professor, Department of Physical Medicine \& Neuro-Rehabilitation, National Institute of Neurosciences \& Hospital, Dhaka

3. Dr. Jebun Nessa, Associate Professor, Department of Physical Medicine \& Rehabilitation, Shaheed Suhrawardy Medical College, Dhaka

4. Dr. Md. Sk Shahidullah, Assistant Professor, Department of Microbiology, National Institute of Cardiovascular Disease, Dhaka

5. Dr. Shahina Sobhan, Associate Professor, Department of Biochemistry, National Institute of Cardiovascular Disease, Dhaka

6. Dr. Shahanaz Begum, Professor, Department of Anatomy, National Institute of Cardiovascular Disease, Dhaka

\section{Correspondence}

Dr. Fahmida Hafez, Assistant Professor \& Head, Department of Physical Medicine \& Rehabilitation, National Institute of Cardiovascular Disease, Dhaka, Sher-E-Bangla Nagar, Dhaka, Bangladesh; Email: fahmida.hafez@yahoo.com; Cell no.: +8801715043704

Conflict of interest: No conflict of interest

Contributions by authors: FH, MZI \& JN were diagnosed and managed the patient. FH, MSS, SS \& SB prepared the manuscript and revised the paper. 
occur in conjunction with early onset generalized dystonia which is associated with the DYT1 gene ${ }^{4}$.

\section{Case Report}

A 48 years old male patient attended the outpatient department of physical Medicine \& Rehabilitation at NICVD, with the complaints of slight pain in neck one side with deviation of chin to the right shoulder for 1 year. Initially it was occasional \& mild but now the symptoms increased in severity. Patient could not hold his neck properly \& it makes his neck turns to the right shoulder. Sometime he struggles with head position, resisting but never overcoming the tendency of his head to assume an unnatural posture which prevents the normal daily life activities and makes the life miserable. Previously he was treated as a case of cervical spondylosis by doing cervical spine X-ray where mild osteophytic lipping, were seen in C4,5 and C6,7 level. On physical examination, the patients chin turns towards right shoulder \& his head turns towards left posteriorly. His sternocleidomastoid muscle (SCM) of left side was prominent \& hard. There was also difficulty to keep face forward \& look to the left without holding it. But right SCM muscle was normal .Sensation was normal. Neurological examination revealed no abnormality except mild weakness of right thumb. Routine biochemical investigations were done in addition to copper analysis in urine which were found normal. MRI of brain and neck was done, which were normal except mild cortical atrophy \& mild degenerative change of cervical spine. EMG of neck muscle shows increased activity of left sternocleidomastoid and right splenius capitis muscle. There was also increased activity of right semispinalis capittis. Based on history, physical examination, different imaging modalities \& EMG (Electromyelogram), a diagnosis of cervical dystonia was confidently made. The patient was trying to manage accordingly by the drugs, physical therapy and orthosis. To relieve the pain, oral analgesic was used. Different muscle relaxant like baclofen, tizanidine etc. different antipsychotic drug like haloperidol, clonazepam etc, anti-Parkinson drug like carbamazepine, were tried to relieve muscle spasm \& dystonia. Sometimes it decreases the intensity of dystonia $\&$ pain subsided. Different exercise such as strengthening exercise, range of motion exercise, \& stretching exercise were prescribed to reduce the dystonia. The patient was advised to wear a cervical collar during journey \& work. Along with changing daily activities \& training in stress management technique. The judicious use of botulinum toxin was tried twice directly into the affected neck muscle. But all these procedures were failed in this case with little recovery of his cervical dystonia.

\section{Discussion}

Cervical dystonia is a focal dystonia characterized by neck muscles contraction involuntarily-causing abnormal movement and awkward posture of the head and neck. It is a rare disorder that can occur at any age even infancy.
Cervical dystonia most often occurs in middle aged people woman more than men. In most case, the exact cause of cervical dystonia (C/D) in usually unknown ${ }^{6}$. The condition appears sporadically in the absence of a documented family history of the disease. In about $10-15 \%$ of cases more than one family member may be affected 4 . Several families have been attributed with autosomal dominant. Initial symptoms of $\mathrm{C} / \mathrm{D}$ are usually mild. Over time involuntary spasm of the neck muscles will increase the frequency and strength until it reaches a plateau ${ }^{7}$. Symptoms can worsen during stress. Both agonist and antagonist ${ }^{5}$ muscle contract simultaneously during dystonic movement. Usually muscle involved contralateral sternocleidomastoid and ipsilateral splenius capitis muscle ${ }^{5}$. Other symptoms include muscle hypertrophy, neck pain, dysarthria and tremor Physical therapy ${ }^{8}$ helps with some of the movement of the head and neck. By stretching out the muscles that spasm it decreases the length and severity of the attacks. Strengthening exercise is also great for easing pain ${ }^{9-11}$.

The most common treatment for cervical dystonia is the use of botulinum toxin type $\mathrm{A}^{12}$. This paralyzing agent can be injected directly into the neck muscles affected by cervical dystonia. Most people with cervical dystonia seen an immediate improvement with this treatment but may be repeated every 3-4 months ${ }^{1-2}$. In this case even botulinum toxin was failed. There is no cure for cervical dystonia. In some people signs and symptoms may disappear without treatment. But recurrence is common. Even surgery like deep brain stimulation may be required to repair the affected muscles.

\section{Conclusion}

Physiatric intervention plays an important role by exercises that improve neck strength and flexibility to aid the patient in keeping their head in proper alignment with their body. Judicious use of a neck brace and training in stress management technique also help a lot. The disability and pain caused by cervical dystonia may result in depression. A high index of clinical suspicion is required for the early diagnosis of cervical dystonia and avoid missing the potentially life miserable condition.

\section{References}

1. Geyer HL, Brenman SB. The diagnosis of dystonia. Lancet Neurology 2006;5(9):780-790

2. Van Herwaarden G, Anten HWM, Hoogduin CAL, Niewold JUR, Roos RAC, Speelman JD, Van Weerden TW, Horstink M. Idiopathic spasmodic torticollis: a survey of the clinical syndromes and patients' experiences. Clinical Neurology and Neurosurgery 1994;96(3):222-225

3. de Carvalho Aguiar PM, Ozelius LJ. Classification and genetics of dystonia. The Lancet Neurology 2002;1(5):316-325

4. Waddy HM, Fletcher NA, Harding AE, Marsden CD. A genetic study of idiopathic focal dystonias. Annals of Neurology 1991; 29(3):320-324

5. Ochudio S, Drzyzga K, Drzyzga LR, Opala G. Various patterns of guests antagonists in cervical dystonia. Parkinsonism and Related Disorders 2007;13(7):417-420 
6. Richter A, Lascher W. Pathophysiology of idiopathic dystonia: findings from genetic animal models. Progress in Neurobiology 1998;54(6):633677

7. Velickovic M, Benabou R, Brin MF. Cervical dystonia pathophysiology \& treatment options. Drugs 2001;61(13):1921-43

8. Crowner BE. Cervical dystonia: disease profile and clinical management. Physical Therapy 2007;87(11):1511-1526

9. Adam OR, Jankovic J. Treatment of dystonia. Parkinsonism Relat Disond 2007;(suppl3): S362-8
10. Silfors A, Goran S. Living with dystonia. La Kartidningen 2002;99(8):786-789

11. Catarina F, Sten-Magnus A, Lindmark B. Physiotherapy in cervical dystonia six experimental single-case studies. Physiotherapy Theory Practice 2008;24(4):275-290

12. Tassorelli C, Francesca M, Laura B, Claudio P. Botulinum Toxin and neuromotor rehabilitation: An integrated approach to idiopathic cervical dystonia. Movement Disorder 2006;21(12):2240-2243 